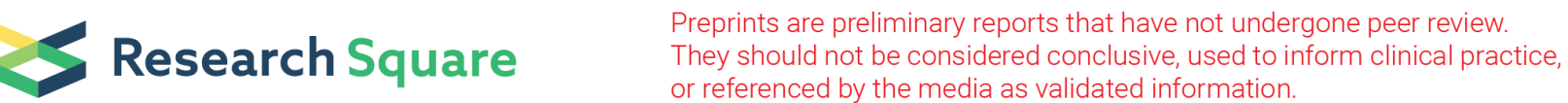

\section{Lactobacillus Reuteri grows in the Microalga Isochrysis Galbana Generating a Fermented Compound that reduces the AIEC Bacteria Harmfulness}

\section{E. Colantoni}

Sapienza University of Rome

\section{F. Palone}

\section{ENEA}

V. Cesi

ENEA

B. Leter

ENEA

G. Sugoni

ENEA

\section{Laudadio}

Sapienza University of Rome
A. Negroni

ENEA

R. Vitali

ENEA

L. Stronati ( $D$ laura.stronati@uniroma1.it )

Sapienza University of Rome

\section{Research Article}

Keywords: galbana, reuteri, aiec, obtained, cells, microalgae, omega3, probiotics

Posted Date: June 4th, 2021

DOl: https://doi.org/10.21203/rs.3.rs-562224/v1

License: (c) (1) This work is licensed under a Creative Commons Attribution 4.0 International License. Read Full License 


\section{Abstract}

Microalgae are currently considered as alternative sustainable resources for high-value bioproducts such as omega3 polyunsaturated fatty acids (PUFAs). Probiotics are assumed to benefit human health by their direct actions on the composition and function of gut microbiota. Aims of the study are: 1) to set up the anaerobic growth of the probiotic Lactobacillus reuteri (L. reuteri) in the omega3-rich microalga Isochrysis galbana (I. galbana); 2) to assess the potential role of the obtained fermented compound (FC) to control the harmfulness of adherent invasive Escherichia coli (AIEC) to intestinal epithelial cells.

I. galbana powder solubilized in PBS was used for the anaerobic growth of $L$. reuteri. The lipidic content of I. galbana and FC was analyzed by GC-MS. Colorectal adenocarcinoma cells CACO2 and the AIEC strain LF82 were used for in vitro experiments.

I. galbana is shown to be an excellent culture medium for growing $L$. reuteri. The obtained FC significantly reduces the AIEC adhesiveness and invasiveness to intestinal epithelial cells.

We show for the first time that microalgae may represent an innovative culture medium to grow probiotics in anaerobiosis. The obtained FC shows beneficial properties for human health by controlling the harmfulness of AIEC bacteria.

\section{Introduction}

Among functional foods, microalgae are focusing the attention of the whole world thanks to their high content of elements of high value for the well-being of consumers, such as carotenoids, vitamins, essential amino acids, polyphenols and oils rich in omega [1-7]. Several studies have found that many of these components have an important impact on human health as they exhibit anti-mutagenic, anticancer, anti-oxidant and anti-inflammatory effects $[8,9]$. Microalgae rich in polyunsaturated fatty acids (PUFA), in particular omega3-PUFA, which include eicosapentaenoic acid (EPA) and docosahexaenoic acid (DHA), have aroused particular interest. Indeed, these bioactive compounds appear to be strategic for their effects on the cardiovascular system and for the prevention of cognitive disorders [10-14].

Microalgae species that produces high PUFA concentrations are Nanochloropsis gaditana, Isochrysis galbana, Phaeodactylum tricornutum, and Crypthecodinium cohnii [15]. In particular, Isochrysis galbana (I. galbana) can synthesize EPA and DHA and contains vitamins, polysaccharides, sterols and carotenoids which make it a valid source for human and animal nutrition [16-18]. Interestingly, several studies have also shown that I. galbana has beneficial effects on human health and could be thought as a nutraceutical as well as therapeutic agent for the management of inflammatory and oxidative damagederived disorders [19-21].

Therapeutic microbiology is expanding and beneficial bacteria are being implemented as treatment and prevention strategies for immune disorders and infectious diseases. Currently, probiotics have become highly recognized as supplements for humans and animals due to their beneficial outcome on health 
improvement and well-being maintenance [22-25]. Lactobacillus reuteri (L. reuteri), a commensal-derived anaerobic probiotic, that resides in the human gastrointestinal tract, shows many beneficial effects, such as prevention and/or amelioration of diverse disorders [26-29]. Recent evidence highlights the role of $L$. reuteri in controlling the growth and survival of pathobionts correlated with infectious or chronic gastrointestinal diseases, such as the adherent-invasive Escherichia coli (AIEC) [30, 31].

Here, we present an innovative and low-cost method for the growth of L. reuteri in raw seaweed extracts from I. galbana instead of the conventional medium, under conditions of oxygen deprivation (anaerobiosis). We demonstrate for the first time that I. galbana is an excellent culture medium for the growth of $L$. reuteri. Moreover, we show that the omega-3 present in the seaweed are still available after the fermentation process and the fermented compound (FC), obtained from the growth of L. reuteri in $I$. galbana in anaerobiosis and thus consisting of the probiotic in a medium with available microalgaereleased PUFA-omega3, slows the growth of AIEC bacteria and significantly limits their adhesiveness and invasiveness to intestinal epithelial cells.

\section{Materials And Methods}

\section{Microalgal and bacterial strains}

I. galbana lyophilized (Archimede Ricerche Srl, Camporosso, Imola, Italy) was solubilized in phosphate buffered saline (PBS) $(36 \mathrm{mg} / \mathrm{ml})$ and used for bacterial growth.

The adherent-invasive AIEC strain LF82 (kindly provided by Prof. Arlette Darfeuille-Michaud, Clermont Universite, Universite d'Auvergne, Clermont-Ferrand, France) was cultured in Tryptone Soy Agar (TSA; plates Oxoid, Basingstoke, UK) for 24 hours at $37^{\circ} \mathrm{C}$ and then sub-cultured in Tryptone Soy Broth (TSB; Oxoid, Basingstoke, UK) with overnight incubation at $150 \mathrm{rpm}, 37^{\circ} \mathrm{C}$.

Powder of L. reuteri DSM17398 (BioGaia, Stockholm, Sweden) was kept at $-20^{\circ} \mathrm{C}$, inoculated in commercial medium De Man, Rogosa and Sharpe (MRS; Sigma-Aldrich, St. Louis, USA) and incubated overnight, $37^{\circ} \mathrm{C}$ without agitation.

\section{Cell culture}

Human colorectal adenocarcinoma cell line, $\mathrm{CACO} 2$, was obtained from the American Type Culture Collection (ATCC, Rockville, MA, USA). Cells were grown at confluence at $37^{\circ} \mathrm{C}$ in Dulbecco's minimum essential medium (DMEM; Gibco, Life Technologies, Carlsbad, CA, USA), supplemented with 10\% inactivated fetal bovine serum (FBS; Euroclone, Milan, Italy) and $2 \mathrm{mM} \mathrm{L-glutamine,} 100 \mathrm{U} / \mathrm{ml}$ penicillin and $100 \mathrm{~g} / \mathrm{ml}$ streptomycin (Biochrom, Berlin, Germany).

\section{Anaerobic growth of $L$. reuteri}

For anaerobic growth, $2 \times 10^{6} \mathrm{CFU} / \mathrm{ml}$ of $L$. reuteri were inoculated in MRS or in $5 \mathrm{ml}$ of I. galbana solubilized in PBS $(36 \mathrm{mg} / \mathrm{ml})$. Each vial was capped and incubated anaerobically without agitation at 
$37^{\circ} \mathrm{C}$ for 120 hours (5 days).

The bacterial growth was evaluated at different times $(24,48,72,96,120$ hours) by plating serially diluted samples in PBS on MRS agar plates $\left(1,2 \%\right.$ agarose) and incubated at $37^{\circ} \mathrm{C}$ for 24 hours. Resulting colonies were counted and the viability $(\mathrm{CFU} / \mathrm{mL})$ value was calculated based on the plated dilution. After 5 days, the fermentation compound (FC) contained an average concentration of $3.5 \times 10^{7} \mathrm{CFU} / \mathrm{ml}$.

\section{Lipid extraction}

Lipids were extracted from l. galbana $(36 \mathrm{mg} / \mathrm{ml})$ solubilized in PBS and FC of a single experiment and the analysis was performed in duplicate.

Samples were freeze-dried for 2 days at $-40^{\circ} \mathrm{C}$ and $60 \mathrm{mBar}$ pressure by freeze-dryer (Edwards). Each sample (5 mg) was resuspended with $1 \mathrm{ml}$ of dichloromethane (DCM) and $0.5 \mathrm{ml}$ of methanol/sulfuric acid $\left(\mathrm{MeOH} / \mathrm{H}_{2} \mathrm{SO}_{4}\right)$ and sonicated for 1 hour at $50^{\circ} \mathrm{C}, 40 \mathrm{KHz}$ frequency. Hexane $(1 \mathrm{ml})$ was used as extracting solvent and, after agitation, calcium carbonate $(16 \mathrm{mg})$ and $\mathrm{H}_{2} \mathrm{O}(1 \mathrm{ml})$ were added and samples were centrifugated for $5 \mathrm{~min}$ at $2000 \mathrm{rpm}$. The separation of polar from apolar phase was repeated twice and finally the latter was dried with nitrogen flow (4 $\mathrm{ml}$ for each sample).

\section{Gas-chromatography mass-spectrometry (GC-MS)}

GC-MS analysis was performed by a 7890A gas chromatograph (Agilent) with capillary columns SBP2331 (Sigma-Aldrich) [60 m, $0.25 \mathrm{~mm}$ inner diameter (ID), $0.2 \mu \mathrm{m}$ film thickness]. Helium was used as carrier gas at a linear velocity of $36.26 \mathrm{~cm} / \mathrm{sec}$ and $1 \mu$ l of each sample was injected splitless. The initial column temperature was $40^{\circ}$ and held $4 \mathrm{~min}$, ramped to $140^{\circ}$ at the rate of $20^{\circ} / \mathrm{min}$, ramped to $220^{\circ}$ at the rate of $2^{\circ} / \mathrm{min}$ and held $1 \mathrm{~min}$ and then finally increased to $260^{\circ}$ at the rate of $10^{\circ} / \mathrm{min}$ and kept at this temperature for $5 \mathrm{~min}$. The mass spectra were recorded using a $5975 \mathrm{C}$ mass spectrometer (Agilent) in full scan mode from $45-450 \mathrm{~m} / \mathrm{z}$ and $240^{\circ}$. The fatty acids concentration of each sample was determined using the software Xcalibur (Thermo Scientific, Waltham, USA) and 37 Component FAME Mix (Supelco, USA) was used as external standard for calibration.

\section{AIEC adhesion and invasion assay}

Adhesion assay

CACO2 cells were grown on 24-well plates at confluence $\left(3 \times 10^{5}\right.$ cells $)$ and infected with LF82 $\left(3 \times 10^{6}\right.$ $\mathrm{CFU})$, or LF82+ L. reuteri $\left(3.5 \times 10^{6} \mathrm{CFU}\right)$, or LF82 + I. galbana $(100 \mu \mathrm{l})$, or LF82 + FC $(100 \mu \mathrm{l})$ at $37^{\circ} \mathrm{C}$ for 3 hours. To quantify the adherence of LF82, we followed the protocol of A. Darfeuille-Michaud et al. [32]. Briefly, infected cells were washed twice in PBS and lysed for 10 minutes with $0.5 \mathrm{ml}$ of $0.1 \%$ Triton X-100 in PBS buffer. Adherent bacteria were recovered and plated on LB agar plates. The latter were incubated at $37^{\circ} \mathrm{C}$ overnight and then the colonies were counted for statistical analysis. 
Invasion assay

CACO2 cells were infected and incubated as above. For invasion assay, we followed the protocol of A. Darfeuille-Michaud et al [32]. Briefly, after incubation, cells were washed twice in sterile PBS and then incubated in DMEM and McCoy's medium, respectively with $0.1 \mathrm{mg} / \mathrm{ml}$ gentamicin for 1 hour to kill the extracellular bacteria. Cells were washed twice in sterile PBS. Lysis, incubation and counts were performed as in the adhesion assay. To ensure maximum reproducibility, accuracy and statistical significance, adhesion and invasion assays were carried out simultaneously in triplicates. To obtain an accurate count of adhesive bacteria, the number of invasive colonies was subtracted from the number of the adhesive ones.

\section{Statistics}

Data are given as mean \pm standard deviation. All experiments were repeated three times. Comparison between groups was performed by a two-tailed Student t-test (significance taken as $P<0.05$ ).

\section{Results}

The unicellular microalga $I$. galbana is an excellent culture medium for the growth of $L$. reuteri

I. galbana extracts were obtained from dehydrated whole seaweed. L. reuteri was inoculated at a concentration of $2 \times 10^{6} \mathrm{CFU} / \mathrm{ml}$ in physiological solution containing l. galbana $(36 \mathrm{mg} / \mathrm{ml}$ ) or commercial medium (MRS) and placed at $37^{\circ} \mathrm{C}$. The growth was followed for 5 days, that is the time necessary for the bioavailable sugars to guarantee the growth of the lactobacillus in anaerobiosis. Results showed that L. reuteri grows in the microalga medium similarly as in MRS reaching the final concentration of $3.5 \times 10^{7}$ and $1.9 \times 10^{7}$, respectively (Fig. 1 ).

The PUFA-omega3 content of the microalgae I. galbana are still mostly available after the fermentation by L. reuteri

The GC-MS lipidomic analysis of I. galbana confirmed that the microalga is rich in omega3, especially DHA. More interestingly, the analysis showed that the availability of DHA and EPA is similar before and after fermentation by $L$. reuteri. Indeed, the amount of EPA is unchanged, while alpha-linoleic acid (ALA) and DHA undergoes a small variation between 15 and $20 \%$ less after fermentation (Fig. 2). Therefore, the $\mathrm{FC}$, in its fullness, contains the probiotic but is also rich in omega 3 and, thus, might represent a very innovative and beneficial compound for the human health.

\section{I. galbana favors the growth of the probiotic L. reuteri respect to that of the pathobiont AIEC LF82}

The AIEC bacteria, known as potent pro-inflammatory microorganisms, strongly increase in the gut of people with intestinal inflammatory disorders and represent a significant challenge for clinicians. Then, we investigated whether I. galbana could also represent an optimal culture medium for the growth of harmful bacteria. Then, L. reuteri and the AIEC prototype, LF82, were grown together in the microalgae. 
Interestingly, although LF82 had been inoculated at a concentration of 1 log higher, in order to mimic the selective advantage this bacterial group has in the inflamed environment, however, the growth curve of LF82 decreases after the first 24 hours while that of $L$. reuteri improves and at the end of fermentation the two species are present at roughly the same concentration $\left(9.9 \times 10^{6}\right.$ and $6 \times 10^{6}$, respectively), clearly suggesting that the probiotic has an advantage in the microalgae culture medium (Fig. 3).

The FC derived from the 5 days-growth of L. reuteri in I. galbana strongly limits the adhesiveness and invasioness of LF82 to intestinal epithelial cells

The human epithelial colorectal adenocarcinoma cells, $\mathrm{CACO}$, are a recognized in vitro model of intestinal epithelial barrier. Hence, we used confluent CACO2 cells to assess the ability of the FC, administered as such, to control the adhesiveness and invasiveness of AIEC LF82, better that the probiotic alone. Confluent CACO2 were exposed for 3 hours to LF82 alone $\left(3 \times 10^{6} \mathrm{CFU}\right)$ or LF82 + L. reuteri $\left(3,5 \times 10^{6} \mathrm{CFU}\right)$ or LF82 + I. galbana $(100 \mu \mathrm{l})$ or LF82 + FC $(100 \mu \mathrm{l})$.

Results showed that the FC significantly reduces the adhesion $(P=0.002)$ and invasion $(P=0.0002)$ of LF82 compared to the probiotic alone. Surprisingly, I. galbana shows a very good capacity to decrease AIEC harmfulness even if lower than that of the FC (Fig. 4).

\section{Discussion}

To date, probiotic production has almost exclusively been carried out using conventional batch fermentation and suspended cultures, but there is an emerging interest from the scientific community and increasing demand from the business world to explore and set up innovative fermentation technologies.

Here, we present a new method to grow the probiotic L. reuteri in the microlaga I. galbana, under anaerobiosis condition. The advantages of this protocol are several. First, the cost is low. Besides, the probiotic can be administered without being previously purified from its culture medium, which, on the contrary, being made up of an omega3-rich microalga, could show beneficial properties for the host organism. Finally, since probiotics must colonize an oxygen-deprived gut environment, the fermentation of the microalga in anaerobiosis can be considered a form of pre-adaptation of probiotics that could improve their survival in the bowel.

Interestingly, our results show for the first time that the microalgae I. galbana is an excellent medium to grow the probiotic L.reuteri, which in fact lives and proliferates in the microalgae as in the conventional medium.

Recent data demonstrate that adding the microalgae Chorella vulgaris to the Lactobacillus spp. growth medium accelerates the growth and metabolic activity of bacteria, suggesting that the combination allows for the creation of innovative, functional products which confer favorable properties to the final product [33]. 
In addition to proposing the microalgae as a growth medium for Lactic acid bacteria, we also support the idea that the microalgae-probiotic combination shows great potential for generating a novel functional product. Actually, we show that, after fermentation, the PUFA-omega3, of which Isochrysis is rich, are not consumed by $L$. reuteri, who instead prefers the consumption of sugars, and remain almost all available in the FC, giving the latter beneficial properties.

Accordingly, we aimed to investigate whether the FC had the ability to impair AIEC-induced inflammation with greater effectiveness than the probiotic alone. AIEC bacteria represent a particular pathotype of Escherichia coli abnormally colonizing the intestinal mucosa of patients with chronic intestinal inflammation, such as those affected by Crohn's disease [34]. Recent evidence suggests that perturbation of the microbial community (dysbiosis) favors the emergence of opportunistic pathogens, in particular AIEC, that can increase the incidence and severity of gut inflammation, since they strongly adhere to and invade intestinal epithelial cells, inducing inflammatory cytokine secretion [35]. Using an in vitro model of gut epithelial barrier, we first confirmed previous evidence that $L$. reuteri is able to reduce the pathogenicity of enteroinvasive Escherichia coli [36, 37], including AIEC [31]. More interestingly, we showed that the treatment with FC prevented the AIEC adhesiveness and invasiveness to epithelial cells more effectively than the probiotic alone. We speculate that this improved effect is due to the omega3 present in the compound that act in combination with the probiotic.

Furthermore, we showed for the first time that I. galbana itself significantly reduces the adhesiveness and invasiveness of AIEC LF82 to epithelial cells. Although previous authors demonstrated that several microalgae, including I. galbana, are prospective candidates to inhibit the growth of gram-positive bacteria [38], however, to our knowledge, their potential in controlling pathobionts has never been reported yet. It is worth noting that in our experimental set up, I. galbana had already proven to be a deterrent for the AIEC group, hampering their growth while favoring that of $L$. reuteri.

\section{Conclusions}

Current evidence indicates that microalgae have the potential to become a novel source of bioactive molecules, especially for those who might wish to enhance the nutritional and functional quality of foods. Even further, we show that microalgae may represent an innovative culture medium to grow probiotics in anaerobiosis and that the obtained FC should be administered as such without separating the probiotic from the culture medium which, indeed, by selecting a proper microalga, could be a useful resource of beneficial bioactive molecules such as omega3. Really, we demonstrate that the $\mathrm{FC}$ has beneficial properties by decreasing the harmfulness of AIEC bacteria to gut epithelial cells.

These findings are important in the development of novel tools for the production of probiotics, whose market is growing dynamically, as well as for the supply of novel fermented products that should represent in the future safe therapeutic agents to be utilized for the management of oxidative damagederived or inflammatory disorders. 
Finally, the L. reuteri grown in I. galbana should be considered a true novel vegetarian probiotic since free from all animal-derived ingredients differently from probiotics grown in the traditional culture medium.

\section{Declarations}

\section{Data Availability}

The data that support the findings of this study are available from the corresponding author [L. S.], upon reasonable request.

\section{Author contributions}

S.L. and C.V. conceived and designed the experiments; C.E., S.G. and L.B. conducted the experiments; L.I., V.R., N.A. and P.F. analyzed the data and performed the statistical analysis; S.L. wrote the manuscript. All authors read and approved the final manuscript.

\section{Additional information}

Correspondence and requests for materials should be addressed to S.L.

\section{References}

1. Pereira, A. G. et al. Xanthophylls from the Sea: Algae as Source of Bioactive Carotenoids. Mar Drugs 19, (2021).

2. Barkia, I., Saari, N. \& Manning, S. R. Microalgae for High-Value Products Towards Human Health and Nutrition. Mar Drugs 17, (2019).

3. Galasso, C. et al. Microalgal Derivatives as Potential Nutraceutical and Food Supplements for Human Health: A Focus on Cancer Prevention and Interception. Nutrients 11, (2019).

4. Giordano, M. \& Wang, Q. Microalgae for Industrial Purposes. in Biomass and Green Chemistry: Building a Renewable Pathway (ed. Vaz Jr., S.) 133-167 (Springer International Publishing, 2018). doi:10.1007/978-3-319-66736-2_6.

5. Matos, J., Cardoso, C., Bandarra, N. M. \& Afonso, C. Microalgae as healthy ingredients for functional food: a review. Food Funct 8, 2672-2685 (2017).

6. Makri, A. et al. Lipid synthesized by micro-algae grown in laboratory- and industrial-scale bioreactors. Engineering in Life Sciences 11, 52-58 (2011).

7. Raheem, A., Prinsen, P., Vuppaladadiyam, A. K., Zhao, M. \& Luque, R. A review on sustainable microalgae based biofuel and bioenergy production: Recent developments. Journal of Cleaner Production 181, 42-59 (2018).

8. Kiran, B. R. \& Venkata Mohan, S. Microalgal Cell Biofactory-Therapeutic, Nutraceutical and Functional Food Applications. Plants (Basel) 10, (2021). 
9. Saide, A., Martínez, K. A., lanora, A. \& Lauritano, C. Unlocking the Health Potential of Microalgae as Sustainable Sources of Bioactive Compounds. Int J Mol Sci 22, (2021).

10. Remize, M., Brunel, Y., Silva, J. L., Berthon, J.-Y. \& Filaire, E. Microalgae n-3 PUFAs Production and Use in Food and Feed Industries. Mar Drugs 19, (2021).

11. Chalima, A., Hatzidaki, A., Karnaouri, A. \& Topakas, E. Integration of a dark fermentation effluent in a microalgal-based biorefinery for the production of high-added value omega-3 fatty acids. Applied Energy 241, 130-138 (2019).

12. Aussant, J., Guihéneuf, F. \& Stengel, D. B. Impact of temperature on fatty acid composition and nutritional value in eight species of microalgae. Appl Microbiol Biotechnol 102, 5279-5297 (2018).

13. de Jesus Raposo, M. F., de Morais, R. M. S. C. \& de Morais, A. M. M. B. Health applications of bioactive compounds from marine microalgae. Life Sci 93, 479-486 (2013).

14. Peltomaa, E., Johnson, M. D. \& Taipale, S. J. Marine Cryptophytes Are Great Sources of EPA and DHA. Mar Drugs 16, (2017).

15. Santos-Sánchez, N. F. et al. Lipids rich in $\omega-3$ polyunsaturated fatty acids from microalgae. Appl Microbiol Biotechnol 100, 8667-8684 (2016).

16. Señoráns, M., Castejón, N. \& Señoráns, F. J. Advanced Extraction of Lipids with DHA from Isochrysis galbana with Enzymatic Pre-Treatment Combined with Pressurized Liquids and Ultrasound Assisted Extractions. Molecules 25, (2020).

17. Chacón-Lee, T. L. \& González-Mariño, G. E. Microalgae for 'Healthy' Foods-Possibilities and Challenges. Compr Rev Food Sci Food Saf 9, 655-675 (2010).

18. Gilbert-López, B. et al. Downstream processing of Isochrysis galbana: a step towards microalgal biorefinery. Green Chem. 17, 4599-4609 (2015).

19. Matos, J. et al. Bioprospection of Isochrysis galbana and its potential as a nutraceutical. Food Funct 10, 7333-7342 (2019).

20. Rodríguez-Luna, A. et al. Topical Application of Glycolipids from Isochrysis galbana Prevents Epidermal Hyperplasia in Mice. Mar Drugs 16, (2017).

21. Sun, Y., Wang, H., Guo, G., Pu, Y. \& Yan, B. The isolation and antioxidant activity of polysaccharides from the marine microalgae Isochrysis galbana. Carbohydr Polym 113, 22-31 (2014).

22. Daliri, E. B.-M., Ofosu, F. K., Xiuqin, C., Chelliah, R. \& Oh, D.-H. Probiotic Effector Compounds: Current Knowledge and Future Perspectives. Front Microbiol 12, 655705 (2021).

23. Chang, C.-J. et al. Next generation probiotics in disease amelioration. J Food Drug Anal 27, 615-622 (2019).

24. Sanders, M. E., Merenstein, D. J., Reid, G., Gibson, G. R. \& Rastall, R. A. Probiotics and prebiotics in intestinal health and disease: from biology to the clinic. Nat Rev Gastroenterol Hepatol 16, 605-616 (2019).

25. Zommiti, M., Chikindas, M. L. \& Ferchichi, M. Probiotics-Live Biotherapeutics: a Story of Success, Limitations, and Future Prospects-Not Only for Humans. Probiotics Antimicrob Proteins 12, 1266- 
1289 (2020).

26. Athalye-Jape, G., Rao, S. \& Patole, S. Lactobacillus reuteri DSM 17938 as a Probiotic for Preterm Neonates: A Strain-Specific Systematic Review. JPEN J Parenter Enteral Nutr 40, 783-794 (2016).

27. Mangalat, N. et al. Safety and tolerability of Lactobacillus reuteri DSM 17938 and effects on biomarkers in healthy adults: results from a randomized masked trial. PLoS One 7, e43910 (2012).

28. Mu, Q., Tavella, V. J. \& Luo, X. M. Role of Lactobacillus reuteri in Human Health and Diseases. Front Microbio/ 9, 757 (2018).

29. Sung, V. et al. Lactobacillus reuteri to Treat Infant Colic: A Meta-analysis. Pediatrics 141, (2018).

30. Costanzo, M. et al. Krill oil, vitamin D and Lactobacillus reuteri cooperate to reduce gut inflammation. Benef Microbes 9, 389-399 (2018).

31. Van den Abbeele, P. et al. Arabinoxylans, inulin and Lactobacillus reuteri 1063 repress the adherentinvasive Escherichia coli from mucus in a mucosa-comprising gut model. NPJ Biofilms Microbiomes 2, 16016 (2016).

32. Darfeuille-Michaud A. et al. High prevalence of adherent-invasive Escherichia coli associated with ileal mucosa in Crohn's disease. Gastroenterology 127, 412-421 (2004).

33. Ścieszka, S. \& Klewicka, E. Influence of the Microalga Chlorella vulgaris on the Growth and Metabolic Activity of Lactobacillus spp. Bacteria. Foods 9, (2020).

34. Chervy, M., Barnich, N. \& Denizot, J. Adherent-Invasive E. coli: Update on the Lifestyle of a Troublemaker in Crohn's Disease. Int J Mol Sci 21, (2020).

35. Agus, A., Massier, S., Darfeuille-Michaud, A., Billard, E. \& Barnich, N. Understanding Host-AdherentInvasive Escherichia coli Interaction in Crohn's Disease: Opening Up New Therapeutic Strategies. Biomed Res Int 2014, (2014).

36. Bertin, Y. et al. Lactobacillus reuteri suppresses E. coli 0157:H7 in bovine ruminal fluid: Toward a preslaughter strategy to improve food safety? PLoS One 12, e0187229 (2017).

37. Wang, Y. et al. Probiotic potential of Lactobacillus on the intestinal microflora against Escherichia coli induced mice model through high-throughput sequencing. Microb Pathog 137, 103760 (2019).

38. Alsenani, F. et al. Evaluation of microalgae and cyanobacteria as potential sources of antimicrobial compounds. Saudi Pharm J 28, 1834-1841 (2020).

\section{Figures}




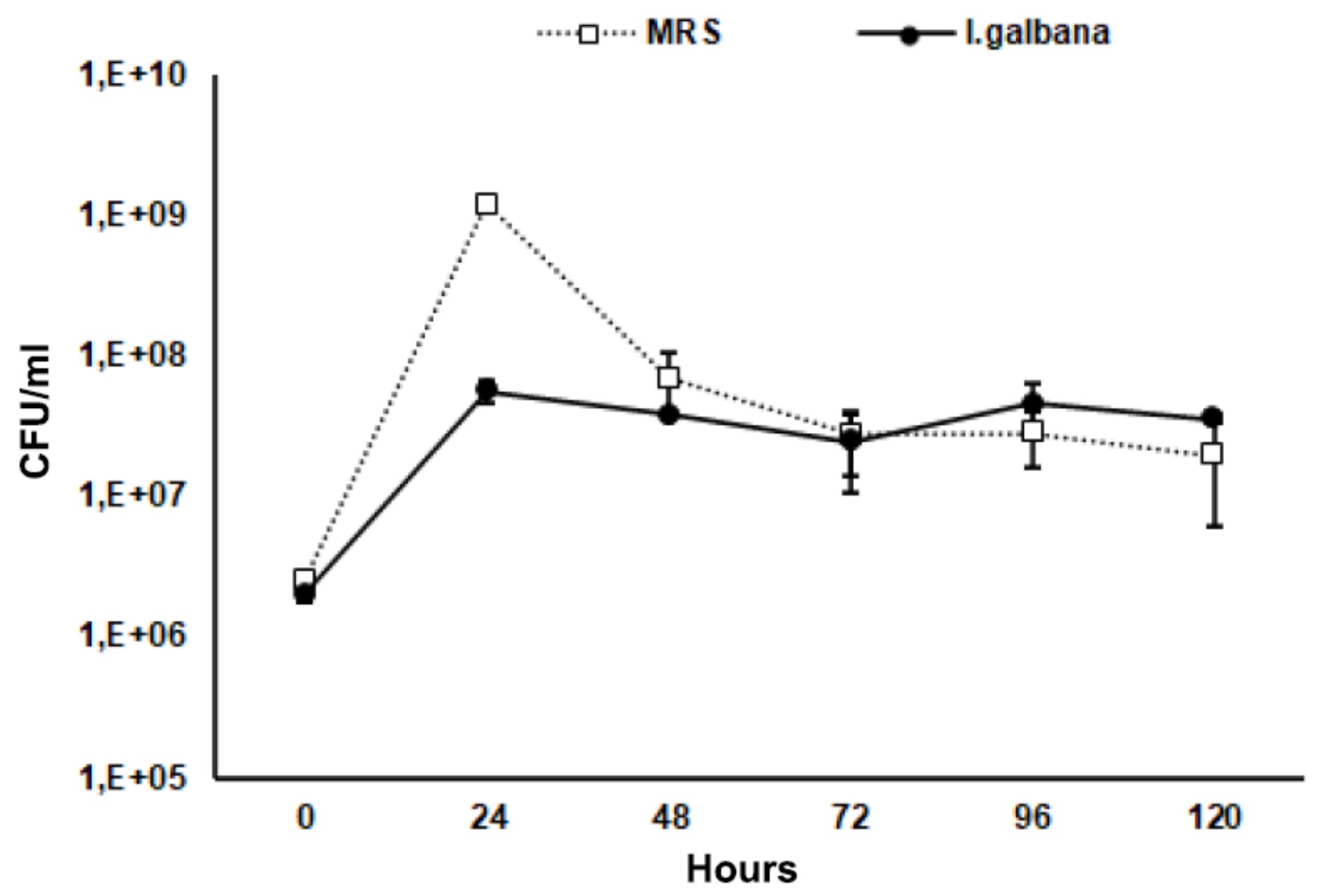

Figure 1

L. reuteri grows well in the microalga I. galbana. L.reuteri reaches the same concentration in I. galbana $(\bullet)$ and MRS ( $\square$ ) after 5 days of fermentation. L. reuteri: Lactobacillus reuteri; I. galbana: Isochrysis galbana; MRS: commercial medium 
- before fermentation a after fermentation
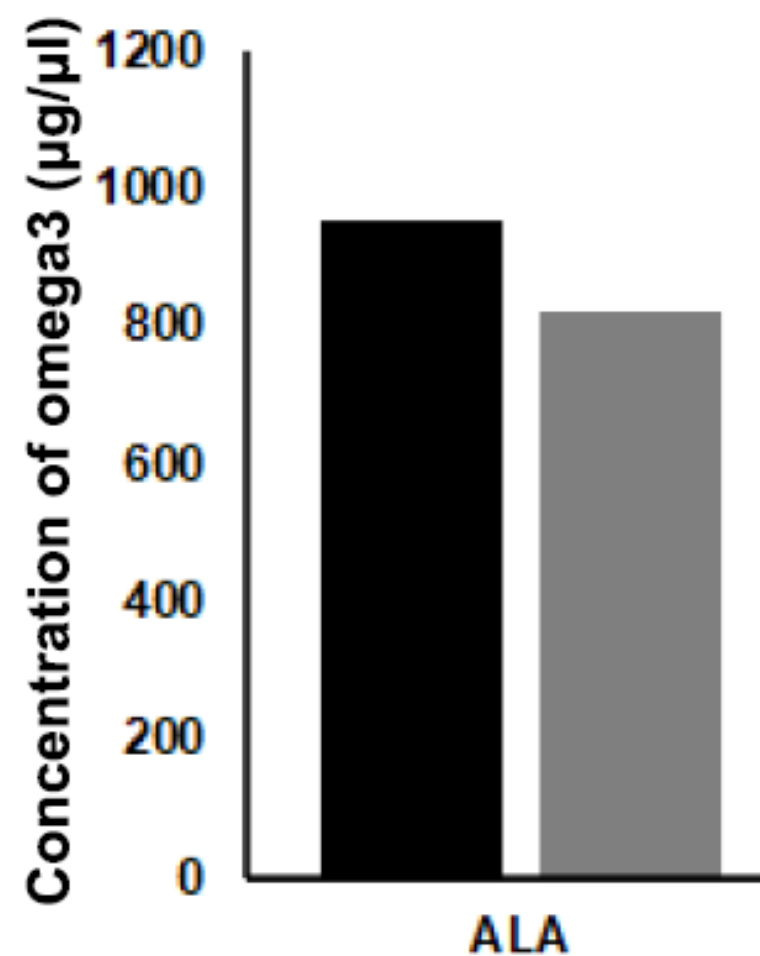

ALA

EPA

DHA

\section{Figure 2}

Microalgal omega3 are still available after the probiotic fermentation. Lipidomic analysis by GC-MS of I. galbana shows that ALA and DHA are slightly reduced, while EPA is unchanged after fermentation. ALA: alpha-linoleic acid; EPA: eicosapentaenoic acid; DHA: docosahexaenoic acid. 


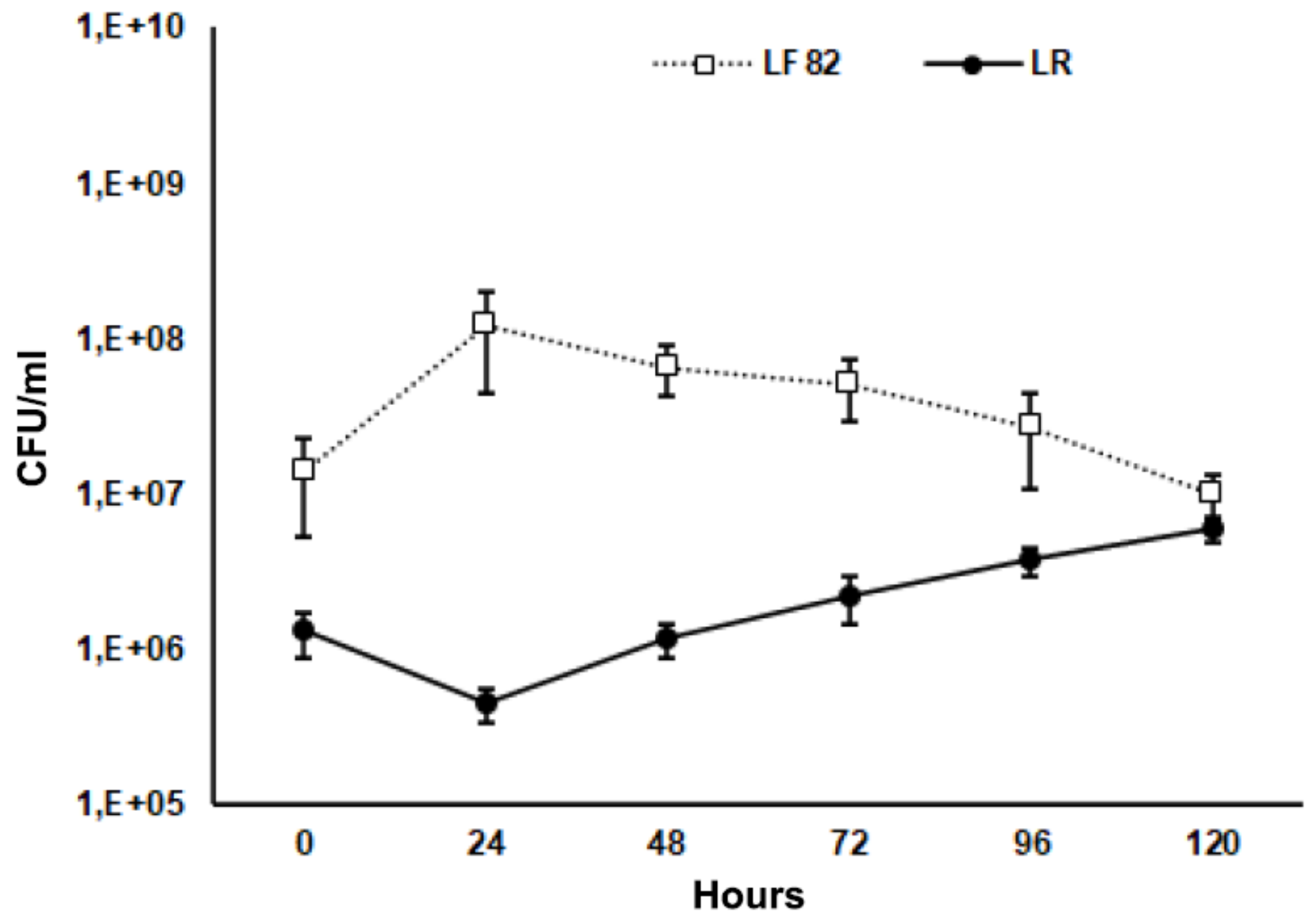

Figure 3

I. galbana favors the growth of L. reuteri respect to AIEC LF82. After 24 hours of growth, LF82 decreases $(\square)$ while L.reuteri increases ( $)$ reaching the same concentration of LF82 after 5 days of fermentation.
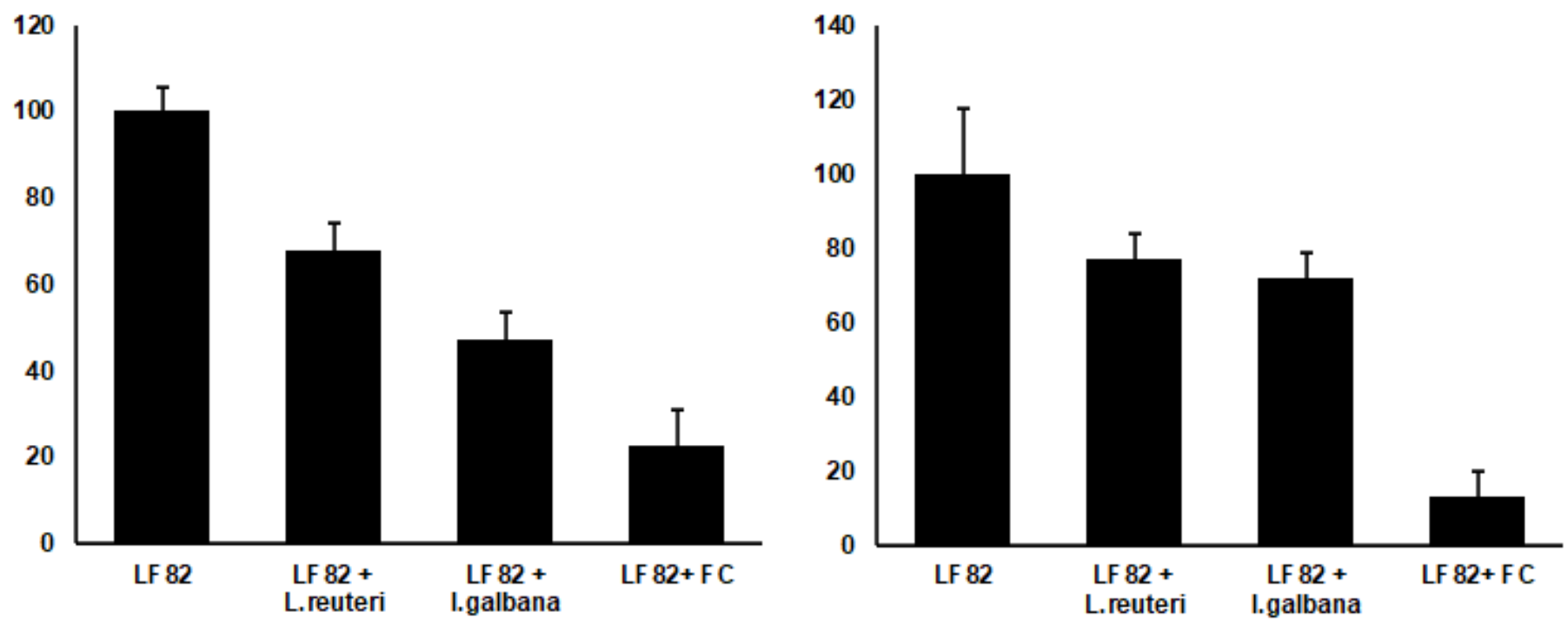


\section{Figure 4}

The FC reduces the adhesiveness and invasiveness of AIEC LF82 to CACO2 cells. The FC significantly reduces the adhesion and invasion of LF82 compared to the probiotic and microalgae alone. AIEC: adherent invasive Escherichia coli; FC: fermented compound. */ $\Delta \mathrm{P}<0.05$; $* \star / \Delta \Delta \mathrm{P}<0,01$; $* \star \star /$ $\Delta \Delta \Delta \mathrm{P}<0,001$. 